\title{
Comparison of the sixth and the seventh editions of the UICC classification for intrahepatic cholangiocarcinoma
}

Benjamin Juntermanns ${ }^{1}$, Gernot Maximilian Kaiser ${ }^{1,2^{*}}$, Lena Orth ${ }^{1}$, Henning Reis ${ }^{3}$, Derar Jaradat ${ }^{1}$, Svenja Sydor ${ }^{4}$, Matthias Buechter ${ }^{4}$, Stefan Kasper ${ }^{5}$, Zoltan Mathé ${ }^{6}$, Georgios Charalambos Sotiropoulos ${ }^{1}$, Hideo Andreas Baba ${ }^{3}$, Ali Canbay ${ }^{4}$, Andreas Paul ${ }^{1}$ and Christian Dominik Fingas ${ }^{1}$

\begin{abstract}
Background: The current seventh edition of the TNM classification for intrahepatic cholangiocarcinoma (ICC) includes tumor number, vascular invasion, lymph node involvement but no longer the tumor size as compared to the sixth edition. The impact of the seventh edition on stage-based prognostic prediction for patients with ICC was evaluated.

Methods: Between 03/2001 and 02/2013, 98 patients with the diagnosis of an ICC were surgically treated at our center. Median survival times were calculated for these patients after separate classification by both sixth and seventh editions.

Results: Median overall survival was increased in patients classified to the lower tumor stages I and II using the seventh as compared to the sixth edition: stage I (54.9 vs. 47.3 months), stage II (19.9 vs. 18.9 months), stage III (17.2 vs. 19.9 months), and stage IV (23.2 vs. 15.3 months), respectively. The seventh edition definition of the T category resulted in an increased median survival regarding the T1 (50.4 vs. 47.3 months) as well as the T2 category (19.9 vs. 15.6 months) and revealed a reduced median survival of patients within the T3 (21.6 vs. 24.8 months) as well as the T4 category (19.9 vs. 27.0 months).

Conclusions: The UICC seventh edition TNM classification for ICC improves separation of patients with intermediate stage tumors as compared to the sixth edition. The prognostic value of the UICC staging system has been improved by the seventh edition.

Trial registration The data for this study have been retrospectively registered and the study has been approved by the ethic committee of the medical faculty of the University Hospital of Essen, Germany (license number 15-6353-BO).
\end{abstract}

Keywords: Cholangiocarcinoma, Intrahepatic cholangiocarcinoma, Liver tumor, Survival, Tumor staging, TNM, UICC

\footnotetext{
${ }^{*}$ Correspondence: gernot.kaiser@st-bernhard-hospital.de

2 Department of General and Visceral Surgery, St. Bernhard-Hospital,

Bürgermeister-Schmelzing-Str. 90, 47475 Kamp-Lintfort, Germany

Full list of author information is available at the end of the article
} 


\section{Background}

Cholangiocarcinoma is a primary cancer of the bile ducts that arises from malignant transformation of bile duct epithelium [1]. This tumor entity can be classified into intrahepatic cholangiocarcinoma (ICC) and extrahepatic tumors (hilar and distal bile duct) depending on its location within the biliary tree [2]. Ten to fifteen percent of all primary liver cancers are ICCs and these neoplasms are the second most common primary liver cancers following hepatocellular carcinomas. Different incidences have been reported ranging from $>80$ per 100,000 population in Thailand to lower incidences in the western world, e.g., Canada with 0.3 per 100,000 population [2-4]. Current studies report that the incidence of ICC is increasing [5, 6]. Comparable to hilar cholangiocarcinoma [7], surgery is the only effective curable treatment up to now $[8,9]$. After surgical therapy, the reported 5-year survival rates range between 30 and 35\% [9-11]. Therefore, a staging system that exactly separates patients suffering from hilar ICC into prognostic groups is desirable to support patient stratification for treatment with regard to future multimodal perioperative therapeutic strategies. The current seventh edition of the American Joint Committee on Cancer (AJCC) staging manual has introduced a new distinct staging system for ICC based on prognostic factors including tumor number, vascular invasion, lymph node involvement but excluded the tumor size in comparison to the sixth edition $[12,13]$. Several studies have focused on the comparison of the sixth and seventh AJCC manual for ICC and found the seventh edition quite suitable for estimation on patients' prognosis [14, 15]. Comparable to our previous hilar cholangiocarcinoma study [7], the purpose of the present work was to compare the prognostic accuracy of the sixth and the new seventh edition of the AJCC staging systems to predict survival after liver resection for ICC in patients treated at the West German Cancer Center, as one of the largest hepatobiliary centers in Germany.

\section{Methods}

\section{Patients and specimens}

Between 03/2001 and 02/2013, 98 patients with the diagnosis of an ICC were surgically treated at our center. This study was conducted in accordance with the Helsinki Declaration of 1975 and approved by the ethic committee of the medical faculty of the University Hospital of Essen, Germany (license number 15-6353-BO). Routine histopathological workup was conducted for all resected tumors by the Department of Pathology of the University Hospital Essen confirming the diagnosis of an ICC. Our cohort included 42 male (42.86\%) and 56 female (57.14\%) patients, with an average age of $62.9( \pm 11.5)$ years. All types of resection margins (R0, R1, and R2) and all cases of irresectable disease were included in the study cohort. Follow-up data were prospectively recorded until February 2014.

\section{Histopathological processing}

Surgical specimens were placed in 4\% neutral-buffered formalin (12-24 h) prior to histopathological processing, dehydrated, and then cleared using an automated standard procedure (Shandon Pathcentre, Thermo Fisher Scientific Inc., USA) before paraffin embedding in Paraplast (McCormick Scientific, USA). From each paraffin block, 3 - to 5- $\mu \mathrm{m}$ sections were cut and Hematoxylin \& Eosin slides were prepared adherent to in-house standards. Histopathology reports were available for every case and included macroscopic and microscopic tumor evaluations, in a continuous text summary, and the epicritical report including the TNM (Classification of Malignant Tumors). Data including operative reports and surgical pathology reports of all patients were entered prospectively into a computer database. Cases were stratified according to the sixth and seventh editions of the AJCC/ UICC (International Union Against Cancer) TNM classification algorithm for ICC $[12,13]$. The sixth and seventh editions of the AJCC/UICC TNM classification algorithm for ICC are compared in Table 1.

\section{Statistical analysis}

Changes in the distribution of TNM classifications and UICC stages were compared between the sixth and seventh editions, and median survival and survival ranges were calculated independently for each classification. Additionally, 1-, 3-, and 5-year survival rates were calculated. Overall survival was defined as the time from the date of surgery to the date of ICC-specific death or the date of last follow-up. Kaplan-Meier survival plots were generated and comparisons made using the log-rank statistic. All parameters that revealed significant results in univariate Kaplan-Meier analysis were subjected to multivariate Cox proportional hazards regression survival analysis to evaluate the prognostic value of the TNM categories and UICC stages derived from the sixth and seventh editions. Differences of $p<0.05$ were considered to be statistically significant.

\section{Results}

A total of 98 patients suffering from ICC were surgically treated at our center between 03/2001 and 02/2013. In this retrospective study, we compared the impact of applying either the sixth or seventh editions of UICC tumor staging to stratify median patient survival or predict prognosis in this patient cohort. A summary of differences between the sixth and seventh editions of UICC staging of ICC and the respective TNM categories is 
Table 1 Sixth and seventh editions of the AJCC/UICC TNM classification algorithm for intrahepatic cholangiocarcinoma (ICC)

\begin{tabular}{|c|c|c|c|c|}
\hline \multirow{2}{*}{$\mathrm{T} 1$} & \multicolumn{2}{|l|}{ Sixth edition } & \multicolumn{2}{|c|}{ Seventh edition } \\
\hline & Solitary tumor without vascular invasion & $\mathrm{T} 1$ & \multicolumn{2}{|c|}{ Solitary tumor without vascular invasion } \\
\hline \multirow[t]{2}{*}{$\mathrm{T} 2$} & \multirow[t]{2}{*}{ Solitary tumor with vascular invasion or multiple tumors, none more than $5 \mathrm{~cm}$} & $\mathrm{~T} 2 \mathrm{a}$ & \multicolumn{2}{|c|}{ Solitary tumor with vascular invasion } \\
\hline & & $\mathrm{T} 2 \mathrm{~b}$ & \multicolumn{2}{|c|}{ Multiple tumors, with or without vascular invasion } \\
\hline T3 & $\begin{array}{l}\text { Multiple tumors more than } 5 \mathrm{~cm} \text { or tumor involving a major branch of the portal } \\
\text { or hepatic veins }\end{array}$ & T3 & \multicolumn{2}{|c|}{$\begin{array}{l}\text { Tumor(s) perforating the visceral peritoneum or } \\
\text { direct extension to extrahepatic structures }\end{array}$} \\
\hline $\mathrm{T} 4$ & $\begin{array}{l}\text { Tumor(s) with direct invasion of adjacent organs other than the gallbladder or with } \\
\text { perforation of visceral peritoneum }\end{array}$ & $\mathrm{T} 4$ & \multicolumn{2}{|c|}{ Tumor with periductal invasion } \\
\hline No & No regional lymph node metastases & No & \multicolumn{2}{|c|}{ No regional lymph node metastases } \\
\hline N1 & Regional lymph node metastases & N1 & \multicolumn{2}{|c|}{ Regional lymph node metastases } \\
\hline M0 & No distant metastases & Mo & \multicolumn{2}{|c|}{ No distant metastases } \\
\hline M1 & Distant metastases & M1 & \multicolumn{2}{|c|}{ Distant metastases } \\
\hline \multicolumn{3}{|c|}{ Stage } & \multicolumn{2}{|c|}{ Stage } \\
\hline । & \multicolumn{2}{|l|}{ T1 NO MO } & । & T1 No Mo \\
\hline$\|$ & \multicolumn{2}{|l|}{ T2 NO MO } & $\|$ & T2 NO MO \\
\hline Illa & \multicolumn{2}{|l|}{ T3 NO MO } & \multirow[t]{3}{*}{ III } & \multirow[t]{3}{*}{ T3 NO MO } \\
\hline 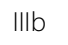 & T4 NO MO & & & \\
\hline IIIC & Any T, N1 M0 & & & \\
\hline \multirow[t]{2}{*}{ IV } & \multirow[t]{2}{*}{ Any T, Any N, M1 } & & IVa & T4 No Mo, Any T N1 Mo \\
\hline & & & $\mathrm{IVb}$ & Any T, Any N, M1 \\
\hline
\end{tabular}

presented in Table $1[12,13]$. We compared the influence of tumor staging using either the sixth or seventh UICC editions on the 1-, 3-, and 5-year survival of patients in this cohort (Table 2).

The median overall survival for patients was staged according to the sixth or seventh editions and broken down by tumor stage: stage I (47.3 or 54.9 months), stage II (18.85 or 19.9 months), stage III (19.9 or 17.2 months), and stage IV (15.3 or 23.15 months), respectively. Staging according to the seventh edition resulted in an increased median overall survival for patients suffering from ICC in the lower UICC tumor stages I and II. A change in tumor staging occurred in 45 out 98 patients $(45.9 \%)$ by using the current classification. We also compared the ability of the sixth and seventh editions of the UICC classification to accurately predict patient prognosis based on UICC tumor stage. Kaplan-Meier survival analysis for patients with different UICC tumor stages revealed that the seventh edition more accurately separates these groups (Fig. 1). The prognostic values of the sixth and seventh

Table 2 Median survival by UICC stage $(n=98)$ using the sixth and seventh editions of the TNM classification

\begin{tabular}{|c|c|c|c|c|c|c|c|}
\hline UICC & $N(\%)$ & $\begin{array}{l}\text { Median survival } \\
\text { in months (range) }\end{array}$ & $\begin{array}{l}\text { Log-rank test ( } p \\
\text { value) }\end{array}$ & $\begin{array}{l}\text { Cox regression } \\
\text { analysis ( } p \text { value) }\end{array}$ & $\begin{array}{l}\text { 1-year } \\
\text { survival (\%) }\end{array}$ & $\begin{array}{l}\text { 3-year } \\
\text { survival (\%) }\end{array}$ & $\begin{array}{l}\text { 5-year } \\
\text { survival } \\
(\%)\end{array}$ \\
\hline 6th edition & & & 0.005 & 0.053 & & & \\
\hline 1 & $43(43.9 \%)$ & $47.30(0.30-149.29)$ & & & 88.4 & 46.5 & 23.3 \\
\hline$\|$ & $8(8.2 \%)$ & $18.85(0.32-97.05)$ & & & 75.0 & 12.5 & 12.5 \\
\hline III & $45(45.9 \%)$ & $19.90(0.16-150.11)$ & & & 64.4 & 28.9 & 8.9 \\
\hline IV & $2(2.0 \%)$ & $15.30(2.70-27.89)$ & & & 50.0 & 0.0 & 0.0 \\
\hline 7th edition & & & 0.006 & 0.082 & & & \\
\hline 1 & $41(41.8 \%)$ & $54.90(0.30-149.29)$ & & & 87.8 & 48.8 & 24.4 \\
\hline$\|$ & $26(26.5 \%)$ & $19.90(0.23-97.10)$ & & & 61.5 & 19.2 & 11.5 \\
\hline III & $3(3.1 \%)$ & $17.20(5.30-43.20)$ & & & 66.7 & 33.3 & 0.0 \\
\hline IV & $28(28.6 \%)$ & $23.15(2.70-150.10)$ & & & 71.4 & 28.6 & 7.1 \\
\hline
\end{tabular}

Survival was calculated using the Kaplan-Meier method and compared using the log-rank test. Cox proportional hazards regression analysis was performed to evaluate the prognostic value of the UICC stage according to the sixth and seventh editions 

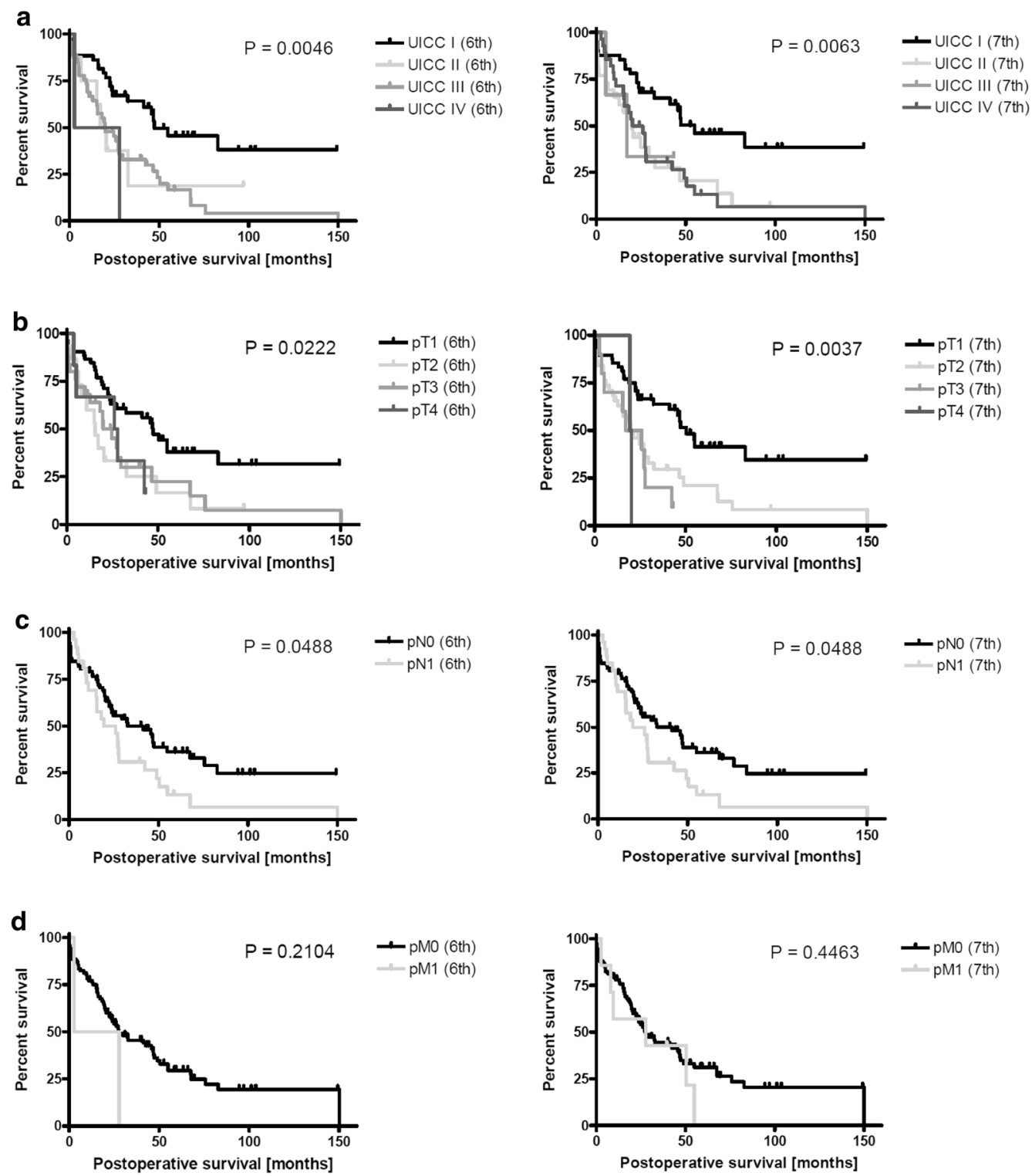

Fig. 1 Comparison of survival prediction ( $n=98$ ) after surgery using the sixth (left) and seventh (right) editions of the UICC tumor classification. Kaplan-Meier analysis was based on tumor stage (a) T category, (b) N category, (c) and M category. (d) Significant survival differences ( $p$ values) were assessed using the log-rank test

editions were confirmed by log-rank test and displayed no relevant differences regarding UICC stages $(p=0.005$ and 0.006, respectively; Table 2).

Regarding the extent of tumor infiltration as reflected by the $\mathrm{T}$ (tumor) category, we compared median survival of patients based on either the sixth or seventh edition $\mathrm{T}$ category classification (Table 3 ). The seventh edition definition of the $\mathrm{T}$ category resulted in increased median survival in the T1 (50.4 vs. 47.3 months) as well as the T2 category (19.88 vs. 15.6 months) and revealed a reduced median survival in patients within the $\mathrm{T} 3$ category ( 21.62 vs. 24.8 months) and the T4 category (19.86 vs. 26.95 months). The prognostic value of the seventh edition $\mathrm{T}$ category staging reached a higher level of significance as compared to the sixth edition T category staging ( $p=0.004$ vs. 0.02 by log-rank test; Table 3 ). Thus, the distribution shift of patients suffering from multiple tumors from T3 category in T2b category in the seventh classification clearly improved patient stratification particularly for this intermediate tumor stage.

Lymph nodes positive for cancer can either be defined as regional spreading of the tumor or as metastases. 
Table 3 Median survival by TNM categories $(n=98)$ using the sixth and seventh editions of the TNM classification

\begin{tabular}{|c|c|c|c|c|c|c|c|}
\hline UICC & $N(\%)$ & $\begin{array}{l}\text { Median survival } \\
\text { in months (range) }\end{array}$ & $\begin{array}{l}\text { Log-rank test } \\
\text { ( } p \text { value) }\end{array}$ & $\begin{array}{l}\text { Cox regression } \\
\text { analysis ( } p \text { value) }\end{array}$ & $\begin{array}{l}\text { 1-year } \\
\text { survival (\%) }\end{array}$ & $\begin{array}{l}\text { 3-year } \\
\text { survival (\%) }\end{array}$ & $\begin{array}{l}5 \text {-year } \\
\text { surviva } \\
\text { (\%) }\end{array}$ \\
\hline Patients edition & & & 0.02 & 0.27 & & & \\
\hline $\mathrm{T} 1$ & $52(53.1 \%)$ & $47.30(0.30-149.29)$ & & & 86.5 & 44.2 & 19.2 \\
\hline $\mathrm{T} 2$ & $15(15.3 \%)$ & $15.60(0.23-97.05)$ & & & 60.0 & 20.0 & 13.3 \\
\hline T3 & $25(25.5 \%)$ & $24.80(0.16-150.11)$ & & & 64.0 & 24.0 & 12.0 \\
\hline $\mathrm{T} 4$ & $6(6.1 \%)$ & $26.95(3.84-43.17)$ & & & 66.7 & 33.3 & 0.0 \\
\hline 7th edition & & & 0.004 & 0.09 & & & \\
\hline $\mathrm{T} 1$ & $48(49.0 \%)$ & $50.40(0.30-149.29)$ & & & 85.4 & 47.9 & 20.8 \\
\hline $\mathrm{T} 2$ & $38(38.8 \%)$ & $19.88(0.16-150.11)$ & & & 63.2 & 23.7 & 13.2 \\
\hline T3 & $10(10.2 \%)$ & $21.62(2.69-43.17)$ & & & 70.0 & 20.0 & 0.0 \\
\hline T4 & $2(2.0 \%)$ & 19.86 (19.38-20.34) & & & 100.0 & 0.0 & 0.0 \\
\hline 6th edition & & & 0.05 & 0.74 & & & \\
\hline NO & $72(73.5 \%)$ & $41.26(0.16-149.29)$ & & & 77.8 & 36.1 & 18.1 \\
\hline N1 & $26(26.5 \%)$ & $22.70(2.69-150.11)$ & & & 69.2 & 30.8 & 7.7 \\
\hline 7th edition & & & 0.05 & 0.74 & & & \\
\hline NO & $72(73.5 \%)$ & $41.26(0.16-149.29)$ & & & 77.8 & 36.1 & 18.1 \\
\hline N1 & $26(26.5 \%)$ & $22.70(2.69-150.11)$ & & & 69.2 & 30.8 & 7.7 \\
\hline 6th edition & & & 0.21 & N/a & & & \\
\hline MO & $96(98.0 \%)$ & $27.79(0.16-150.11)$ & & & 76.0 & 35.4 & 15.6 \\
\hline M1 & $2(2.0 \%)$ & $15.29(2.69-27.89)$ & & & 50.0 & 0.0 & 0.0 \\
\hline 7th edition & & & 0.45 & N/a & & & \\
\hline MO & 91 (92.9\%) & $27.79(0.16-150.11)$ & & & 76.9 & 34.1 & 16.5 \\
\hline M1 & $7(7.1 \%)$ & $27.89(2.69-54.97)$ & & & 57.1 & 42.9 & 0.0 \\
\hline
\end{tabular}

Survival was calculated using the Kaplan-Meier method and compared using the log-rank test. Cox proportional hazards regression analysis was performed to evaluate the prognostic value of the TNM categories according to the sixth and seventh editions

The definition of regional lymph nodes has been modified within the $\mathrm{N}$ (lymph nodes) and $\mathrm{M}$ (metastases) categories of the seventh edition of the UICC classification [13]. The overall impact of a positive lymph node on the extent of the disease is emphasized by the seventh edition, such that involvement of any lymph node results in tumor stage IV which has been a tumor stage III in the sixth edition. Applying the seventh edition resulted in reclassification of five patients as M1 based on histological positive lymph node and, therefore, a reclassification from stage IIIc into stage IVa in the current edition (Table 4). Median survival for patients classified for the $\mathrm{N}$ category using the sixth and seventh editions was also compared (Table 3). Median survival of patients classified N0 did not change (41.26 months) and also remained the same for $\mathrm{N} 1$ (22.7 months). Log-rank test confirmed significance for both editions $(p=0.05)$ regarding the $\mathrm{N}$ status.

Using the sixth or seventh edition descriptions of the $M$ category also did not affect median survival of patients when staged M0 (27.79 months). Due to the upgrade of five patients to M1 because of distal lymph node metastases (M1), survival in the M1 stage of the seventh
Table 4 Patients with lymph node metastasis upstaged from $\mathrm{N} 1$ in the sixth edition to $\mathrm{M} 1$ in the seventh edition of the UICC TNM classification

\begin{tabular}{llll}
\hline $\begin{array}{l}\text { Patients } \\
\text { with positive } \\
\text { lymph nodes }\end{array}$ & $\begin{array}{l}\text { Location of lymph node } \\
\text { infiltration }\end{array}$ & $\begin{array}{l}\text { 6th TNM } \\
\text { edition }\end{array}$ & $\begin{array}{l}\text { 7th } \\
\text { TNM } \\
\text { edition }\end{array}$ \\
\hline Female, 68 years & Celiac artery & $\mathrm{N1}$ & $\mathrm{M} 1$ \\
Female, 55 years & Portocaval & $\mathrm{N} 1$ & $\mathrm{M} 1$ \\
Female, 47 years & Celiac artery & $\mathrm{N} 1$ & $\mathrm{M} 1$ \\
Female, 64 years & Celiac artery, portocaval & $\mathrm{N} 1$ & $\mathrm{M} 1$ \\
Female, 76 years & Caval & $\mathrm{N} 1$ & $\mathrm{M} 1$ \\
\hline
\end{tabular}

classification is prolonged as compared to the sixth classification (27.89 vs. 15.29 months, no significance; Tables 3, 4).

\section{Discussion}

In this single-institution study, we found that the seventh edition of the UICC TNM classification for ICC slightly more accurately stratifies patients suffering from this neoplasm. Particularly, the guidelines for the current 
seventh edition AJCC staging manual has introduced a new distinct staging system for ICC based upon prognostic factors including tumor number, vascular invasion, lymph node involvement but no longer the tumor size as opposed to the sixth edition [12,13]. This redefinition enables a more appropriate patient stratification in our cohort. Another novelty is the new definition of distant lymph node metastases resulting in several tumor stage upgrades in our cohort with a change of median survival regarding UICC tumor stage IV.

Tumor staging according to AJCC/UICC seventh edition was accompanied by an increased survival in tumor stages I and II allowing a more appropriate prognosis stratification. The reason for this was an altered classification of 45 out of 98 patients using the current classification. Thus, a total of 67 (seventh edition) instead of 51 (sixth edition) patients is classified to the lower tumor stages I and II in this study. This finding is consistent with a multicenter study conducted by Ribero et al. [14]. This group also reported that the majority of their patients displayed a T1 or a T2 tumor stage and an UICC/AJCC stage I or II. In our opinion, this new distribution allows a better stratification within our cohort and, therefore, a better selection for adjuvant therapy strategies.

We also found several changes regarding the $\mathrm{T}$ status applying the seventh edition. In our cohort, the new $\mathrm{T}$ categories enabled a more appropriate patient stratification, especially for T1 and T2. For T2, this fact is due to the loss of tumor size evaluation causing a downstaging for multiple tumors from T3 to T2b. Nathan et al. [15] could demonstrate that the tumor size does not correlate with any additional prognostic value by analyzing the survival data of 598 patients following resection for ICC. Thus, they concluded that the sixth edition AJCC/ UICC T classification failed to stratify T2 and T3 cohorts into two distinct prognostic groups. Our data support this finding as the prognostic value of the seventh edition $\mathrm{T}$ category staging reached a higher level of significance as compared to the sixth edition $\mathrm{T}$ category staging ( $p=0.004$ vs. 0.02$)$. Another novelty in the seventh AJCC/UICC staging system is the introduction of a separate classification of the T4 category "periductal tumor infiltration." In this study, only two patients were found to show periductal infiltration. Both patients also displayed positive lymph node metastasis and were therefore staged as AJCC/UICC IVa. Thus, the prognostic significance of this new T4 category remains uncertain in our cohort and needs to be investigated more specifically in upcoming studies with larger patient numbers.

The seventh edition also re-evaluates the presence of regional lymph node involvement regarding the $\mathrm{N}$ and $\mathrm{M}$ classifications emphasizing the importance lymph nodes infiltrated with cancer for UICC tumor staging. This resulted in the reclassification of patients with lymph node metastases into UICC stage IV by the seventh edition (formerly stage III in the sixth edition). Additionally, distal lymph nodes were reclassified in the current classification manual [13]. This novelty affected a total of five patients in our cohort categorized as stage III according to the sixth edition of the TNM classification. Due to a celiac or mesenteric lymph node infiltration (N1), the tumors were upstaged to metastatic (M1, stage IV) disease according to the seventh edition (Table 4). Similar to our previous study of perihilar cholangiocarcinoma [7], this novelty did not significantly affect patient survival comparing both groups (Table 3). Interestingly, the new $\mathrm{M}$ staging in our collective caused a prolonged survival in the M1 group. This finding raises the question, if the upgrade from $\mathrm{N} 1$ to $\mathrm{M} 1$ really mirrors an altered prognosis as intended by the AJCC/UICC. Nevertheless, Farges et al. [16] recommended that a routine lymphadenectomy at the time of surgery for ICC should become the standard of care, which again emphasizes the importance of lymph node involvement.

\section{Conclusion}

In conclusion, based on this study of 98 patients treated for ICC at a single institution, the categorization of UICC tumor stages by the seventh UICC edition enables a better patient stratification than the sixth edition. The current edition emphasizes the importance of lymph node involvement and periductal infiltration instead of tumor size. Thus, the seventh edition more appropriate separates intermediate tumor stages as reflected by the median patient survival for this cohort and confers a higher prognostic value to the tumor stage. This should facilitate the stratification of patients diagnosed with ICC into different risk groups resulting in better customized multimodal perioperative treatment strategies. Our data show that particularly the $\mathrm{T}$ categories of the seventh edition enable a more appropriate prediction of patient survival than the corresponding categories from the sixth edition. A considerable number of patients were staged differently by the seventh edition in the present study. This fact should be considered when comparing previous studies employing the sixth edition with new data from ICC patients. Finally, the T4 category (periductal infiltration) and the reclassification of distant lymph nodes need to be re-evaluated in upcoming studies with larger patient numbers.

\section{Abbreviations}

ICC: intrahepatic cholangiocarcinoma; AJCC: American Joint Committee on Cancer; TNM: Classification of Malignant Tumors; UICC: International Union Against Cancer; T: tumor; N: lymph node; M: metastases. 


\section{Authors' contributions}

$\mathrm{BJ}$ is the first author of the manuscript. He made substantial contribution to the conception and design, analysis and interpretation and collection of the data. GMK made substantial contribution to the conception and design, analysis and interpretation of the manuscript. LO was involved in the interpretation and collection of the data and transferred the tumor data from the 6th to the 7th UICC edition. HR from the Institute of Pathology was involved in the data collection and the final approval of histology data and the last version of the manuscript. DJ has been involved in drafting the manuscript and revising it for its intellectual content. Additionally he was involved in data collection. SS was involved in the collection of data, statistical analyses and interpretation of the data. MB was involved in data collection and interpretation and additionally in the writing process of the manuscript. SK was involved in the follow-up of the patients by seeing them in the outpatient department and therefore in the data collection. Additionally he was involved in the writing process and the final approval of the data and the manuscript. ZM made contributions to the conception and design of the study and collection of the data. GCS made substantial contribution to the design of the study and additionally to the interpretation and collection of the data. HAB from the Institute of Pathology was also involved in the data collection and the final approval of histology data and the last version of the manuscript. AC was involved in the collection and interpretation of the data and also in the statistical analyses. AP is the head of the department and made substantial contribution to the conception and design of the study and also collection of the data. CDF is the senior author of the manuscript. He was responsible for the statistical analyses in most parts and made substantial contribution to the conception and design, analysis and interpretation and collection of the data. Additionally he was substantially involved in the writing process of the manuscript. All authors read and approved the final manuscript.

\section{Author details \\ ${ }^{1}$ Department of General, Visceral and Transplantation Surgery, University Hospital Essen, University Duisburg-Essen, Hufelandstrasse 55, 45147 Essen, Germany. ${ }^{2}$ Department of General and Visceral Surgery, St. Bernhard-Hospital, Bürgermeister-Schmelzing-Str. 90, 47475 Kamp-Lintfort, Germany. ${ }^{3}$ Institute of Pathology, University Hospital Essen, University Duisburg-Essen, Essen, Germany. ${ }^{4}$ Department of Gastroenterology and Hepatology, University Hospital Essen, University Duisburg-Essen, Essen, Germany. ${ }^{5}$ Department of Medical Oncology, University Hospital Essen, University Duisburg-Essen, Essen, Germany. ${ }^{6}$ Department of Transplantation and Surgery, Semmelweis University, Budapest, Hungary.}

\section{Acknowledgements}

The assistance of Jutta Hunke regarding patient follow-up is gratefully acknowledged.

\section{Competing interests}

The authors declare that they have no competing interests.

\section{Availability of data and materials}

The datasets supporting the conclusions of this article are included within the article.

\section{Consent for publication}

Not applicable.

\section{Ethics approval and consent to participate}

This study was conducted in accordance with the Helsinki Declaration of 1975 and approved by the ethic committee of the medical faculty of the University Hospital of Essen, Germany (license number 15-6353-BO).

\section{Funding}

This work was supported by the Grants FI 1630/3-1 (DFG/German Research Foundation; CDF) and IFORES D/107-114400 (CDF).

\section{Publisher's Note}

Springer Nature remains neutral with regard to jurisdictional claims in published maps and institutional affiliations.

Received: 23 June 2016 Accepted: 26 May 2018

Published online: 01 June 2018

\section{References}

1. Lafaro KJ, Cosgrove D, Geschwind JF, Kamel I, Herman JM, Pawlik TM. Multidisciplinary care of patients with intrahepatic cholangiocarcinoma: updates in management. Gastroenterol Res Pract. 2015;2015:860861.

2. Bridgewater J, Galle PR, Khan SA, et al. Guidelines for the diagnosis and management of intrahepatic cholangiocarcinoma. J Hepatol. 2014;60:1268-89.

3. Bragazzi MC, Cardinale V, Carpino G, et al. Cholangiocarcinoma: epidemiology and risk factors. Transl Gastrointest Cancer. 2012;1(21-32):1.

4. Fingas CD, Katsounas A, Kahraman A, et al. Prognostic assessment of three single-nucleotide polymorphisms (GNB3 825C >T, BCL2-938C >A, MCL1-386C > G) in extrahepatic cholangiocarcinoma. Cancer Invest. 2010;28:472-8.

5. McGlynn KA, Tarone RE, El-Serag HB. A comparison of trends in the incidence of hepatocellular carcinoma and intrahepatic cholangiocarcinoma in the United States. Cancer Epidemiol Biomark Prev. 2006;15:1 198-203.

6. Kurita S, Mott JL, Cazanave SC, et al. Hedgehog inhibition promotes a switch from type II to type I cell death receptor signaling in cancer cells. PLoS ONE. 2011;6:e18330.

7. Juntermanns B, Sotiropoulos GC, Radunz S, et al. Comparison of the sixth and the seventh editions of the UICC classification for perihilar cholangiocarcinoma. Ann Surg Oncol. 2013;20:277-84.

8. Simo KA, Halpin LE, McBrier NM, et al. Multimodality treatment of intrahepatic cholangiocarcinoma: a review. J Surg Oncol. 2016;113:62-83.

9. Lang H, Sotiropoulos GC, Sgourakis G, et al. Operations for intrahepatic cholangiocarcinoma: single-institution experience of 158 patients. J Am Coll Surg. 2009;208:218-28.

10. Jonas S, Thelen A, Benckert C, et al. Extended liver resection for intrahepatic cholangiocarcinoma: a comparison of the prognostic accuracy of the fifth and sixth editions of the TNM classification. Ann Surg. 2009;249:303-9.

11. Uenishi T, Kubo S, Yamazaki O, et al. Indications for surgical treatment of intrahepatic cholangiocarcinoma with lymph node metastases. J Hepatobiliary Pancreat Surg. 2008;15:417-22.

12. Sobin LH, Wittekind C. International Union Against Cancer (UICC) TNM classification of malignant tumours. 6th ed. New York: Wiley; 2002.

13. Sobin LH, Wittekind C. International Union Against Cancer (UICC) TNM classification of malignant tumours. 7th ed. New York: Wiley; 2009.

14. Ribero D, Nuzzo G, Amisano M, et al. Comparison of the prognostic accuracy of the sixth and seventh editions of the TNM classification for intrahepatic cholangiocarcinoma. HPB (Oxford). 2011;13:198-205.

15. Nathan H, Aloia TA, Vauthey JN, Abdalla EK, Zhu AX, Schulick RD, Choti MA, Pawlik TM. A proposed staging system for intrahepatic cholangiocarcinoma. Ann Surg Oncol. 2009;16:14-22.

16. Farges $O$, Fuks $D$, Le Treut YP, et al. AJCC seventh edition of TNM staging accurately discriminates outcomes of patients with resectable intrahepatic cholangiocarcinoma: by the AFC-IHCC-2009 study group. Cancer. 2011;117:2170-7. 